\title{
El gobierno venezolano y la política salarial implementada a los profesores universitarios (2000-2018)
}

\section{LUIS E. TORRES-NÚÑEZ}

Profesor titular en la Universidad Nacional Experimental Sur del Lago. candidato a doctor en Educación: políticas públicas y profesión docente, MSc en Gerencia Pública, licenciado en Contaduría Pública. Presidente de la Asociación de Profesores de la Universidad Nacional Experimental Sur del Lago (APUNESUR) y miembro de la Junta Directiva de la Federación de Asociaciones de Profesores Universitarios de Venezuela (FAPUV), ambos cargos en el período 2010-2018.

\section{Keta Stephany}

Profesora asociada en la Universidad Central de Venezuela, doctora en Estudios del Desarrollo, MSc en Planificación, mención política social, licenciada en Letras, miembro principal del Consejo de Escuela de la Escuela de Trabajo Social-ucv y miembro principal del Comité Académico del Doctorado en salud pública de la ucv. Secretaria de Actas, Memoria e Información de FAPUV.

María Cristina Parra-Sandoval

Profesora titular emérita en la Universidad del Zulia. Doctora en Estudios del Desarrollo, MSc en Sociología, licenciada en Sociología. Visiting Fellow en la University of Calgary,

Resumen

El artículo analiza la política salarial impuesta por el gobierno nacional a los profesores universitarios en Venezuela, desde el año 2000 al 2018. Se presentan tres subperiodos de análisis y una reflexión final. La política salarial -estructura del salario, escala del salario, interescala salarial y mecanismo de ajuste del salario- legítima y reconocida por los académicos para el inicio del periodo en estudio estuvo enmarcada en el contenido de las Normas de Homologación, hoy desconocidas por el gobierno. Entre las principales violaciones de la política salarial se evidencia la ruptura de la escala salarial, la modificación de los valores de la interescala y la implementación de otros criterios para el ajuste de los salarios.

Palabras clave

Política salarial; profesores universitarios; universidad venezolana; Normas de Homologación. 


\title{
Venezuelan government and the salary policy implemented for university teachers (2000-2018)
}

\begin{abstract}
This article is an analysis of the salary policy imposed by the national government on university professors in Venezuela, from 2000 to 2018, presented in three sub-periods of study and a final reflection. The salary policy -salary structure, salary scale, interscale wage and wage adjustment mechanism- legitimate and recognized by academics for the beginning of the period under study, was confined with the Homologation Norms, unrecognized by the government today. Among the main violations of the salary policy, there is evidence of a salary scale breakup, a modification of interscale values and the implementation of other criteria for the wage adjustment.
\end{abstract}

Keywords

Salary policy; University teachers; Venezuelan University; Homologation Norms.

\section{O governo venezuelano e a política salarial implementada para os professores universitários (2000-2018)}

\section{Sumário}

O artigo analisa a política salarial imposta pelo governo nacional para os professores universitários na Venezuela, desde o ano 2000 até o 2018. Se apresentam três subperíodos de análise e uma reflexão final. A política salarial -estrutura do salário, escala do salário, interescala salarial e mecanismo de ajustamento do salário- legítima e reconhecida pelos acadêmicos para o início do período em estudo, esteve enquadrada no conteúdo das Normas de Homologação, hoje desconhecidas pelo governo. Entre as principais violações da política salarial se manifesta a ruptura da escala salarial, a modificação dos valores da interescala e a implementação de outros critérios para o ajustamento dos salários.

Palavras-chave

Política salarial; professores universitários; universidade venezuelana; Normas de Homologação. 


\section{Introducción}

Uno de los indicadores más relevantes de la expansión de la educación superior que caracterizó la segunda mitad del siglo Xx fue el cambio que se produjo no solo en las funciones cumplidas por el profesor universitario, sino también en sus condiciones laborales. El profesor universitario dejó de ser un catedrático que se distinguía por su dominio de una disciplina en particular y hacía de la docencia la pasión de su vida, para ser un asalariado, con todo lo que ello implica. En Venezuela el proceso no fue ajeno a estas características. El crecimiento y la diversificación de la educación superior fue un proceso mantenido en el tiempo, desde finales de la década del cincuenta, en correspondencia con el inicio del periodo democrático iniciado en 1958. Así, en un poco más de dos décadas -de 1958 a comienzo de los ochenta- la educación superior venezolana tuvo una tasa total de crecimiento de la matrícula de $1.486 \%$, al tiempo que el porcentaje de la población comprendida entre los 20 y 24 años con algún tipo de contacto con este nivel de educación alcanzara a ser el $18.1 \%$. A ello se añade que el número de instituciones de educación superior ascendiera de 12 en 1958 a 83 en 1982. En consecuencia, también se produjo en las instituciones de educación superior un aumento muy considerable en el número de plazas para profesores, expresado en una tasa total de crecimiento de $1.615 \%$ en 1982 en comparación con 1958 (Parra, 1998).

Este crecimiento exponencial del número de profesores universitarios es una evidencia cuantificable del cambio que se produjo en las condiciones laborales de este importante sector. Estas condiciones cada vez estaban más alejadas de la concepción idílica de lo que era ser profesor universitario, en la cual prevalecía su vocación de servicio, sin tener como motivo previo la recompensa económica a través del salario y otros beneficios socioeconómicos. En esta nueva dinámica, y como un indicador del proceso de cambios que se dieron en la universidad venezolana, la figura tradicional del profesor como catedrático es paulatinamente sustituida por el profesor, quien tiene entre sus funciones la docencia, pero para quien se contemplan también obligaciones como investigador, con todo lo que ello implicó en términos de su relación laboral con la institución (Parra-Sandoval, 2003).

Por otra parte, en Venezuela como en la mayoría de los países latinoamericanos, a pesar del reconocimiento explícito, incluso en la normativa legal, de la condición de autonomía otorgada a las universidades, estas han mantenido una conexión con el Estado caracterizada por su dependencia económica, en tanto la mayor parte de su financiamiento proviene de los fondos públicos derivados del presupuesto nacional. Entre 1958 y 1982 esta relación fue de "buena voluntad" entre el Estado y las 
instituciones de educación superior, a pesar de los enfrentamientos que se dieron por razones políticas entre las universidades y los diferentes gobiernos. En este escenario, la universidad participaba de la competencia en la distribución de la renta petrolera, lo cual condujo a la institución a convertirse en fuente de empleo y generadora de beneficios socio económicos para quienes formaban parte del cuerpo académico. Esta situación se deterioró a partir de los años ochenta en virtud del desmejoramiento de la situación económica del país. Este deterioro económico se acentuó a partir de 1982 con la devaluación de la moneda, lo cual llevó a que la relación entre el Estado y las universidades dejara de ser benevolente para entrar en una lucha por obtener del Estado mayores recursos como una constante de la vida universitaria.

Ante este constante enfrentamiento entre las universidades y el Estado por mayores recursos, destinados en un porcentaje importante al pago de salarios y beneficios socio económicos al personal académico, administrativo y de servicios, el gobierno nacional decretó las Normas de Homologación, las cuales constituyen el primer instrumento legal de carácter nacional para regir los ajustes salariales del profesorado universitario desde 1982 . Hasta ese momento, el presupuesto asignado para cubrir los salarios y otros beneficios socioeconómicos otorgados a los profesores era atribución del Consejo Universitario respectivo de cada institución.

La medida, hasta cierto punto adoptada de manera unilateral por parte del gobierno en ese momento, enfrentó a este con las autoridades de las universidades, quienes consideraron que era una forma de coartar la autonomía universitaria y, al mismo tiempo, estimaron que su capacidad de negociación interna con los profesores era disminuida. Su aplicación trajo como consecuencia que se profundizara el enfrentamiento de las instituciones con el gobierno, lo cual condujo a constantes paralizaciones de actividades.

Este mecanismo establece en su artículo 13 el ajuste del salario de los académicos cada dos años, tomando como referencia el índice acumulado de precios al consumidor en ese período (IPC) según cifras del Banco Central de Venezuela. Para ello, las normas contemplan que la negociación entre la Federación de Profesores Universitarios de Venezuela (FAPUV) en representación de los profesores univer- 
sitarios y una comisión del Consejo Nacional de Universidades produzca el acuerdo de aprobación definitiva del salario y demás beneficios de previsión social y salud.

Posteriormente, fueron incorporados a las negociaciones los gremios y sindicatos de empleados y obreros de las universidades, y los de profesores, empleados y obreros de los Institutos y Colegios Universitarios. Así, en un cambio radical de la dinámica, FAPUV ha tenido que jugar un papel fundamental para exigir al Estado el estricto cumplimiento de las Normas de Homologación, toda vez que la economía nacional se adentró, en distintos momentos, en profundas espirales inflacionarias con el consiguiente deterioro de las condiciones salariales de los profesores universitarios. ${ }^{1}$

A lo anteriormente señalado con relación a la aplicación de las Normas de Homologación hay que añadir que toda política pública salarial en Venezuela debería regirse por los postulados contenidos en la norma constitucional vigente (1999), la cual señala en su artículo 91 que el trabajador tendrá “derecho a un salario suficiente que le permita vivir con dignidad y cubrir para sí y su familia las necesidades básicas materiales, sociales e intelectuales..." En este artículo de la Constitución se evidencia coherencia con las disposiciones internacionales establecidas en el artículo 23, numeral 3 de la Declaración Universal de los Derechos Humanos de las Naciones Unidas, refrendada por la Organización Internacional del Trabajo (OIT) en su Conferencia Internacional número 79 de fecha 1992.

En este sentido y, teniendo en cuenta estos antecedentes, en el artículo se exponen y analizan aspectos relevantes de la relación entre el Estado venezolano y los profesores universitarios, con énfasis en la política salarial impuesta por el gobierno durante más de una década, en desconocimiento de la normativa vigente. Tal desconocimiento pareciera tener como objetivo lograr la sustitución de la plataforma académica crítica existente en las principales universidades públicas del país, en detrimento de su autonomía.

La crisis humanitaria por la cual atraviesa Venezuela afecta por igual a todos los sectores de la población, lo cual hace que el análisis de la política salarial de los académicos venezolanos, principalmente desde el año 2000 hasta la fecha, sea complejo. En el artículo se trata de analizar la política salarial referida a 


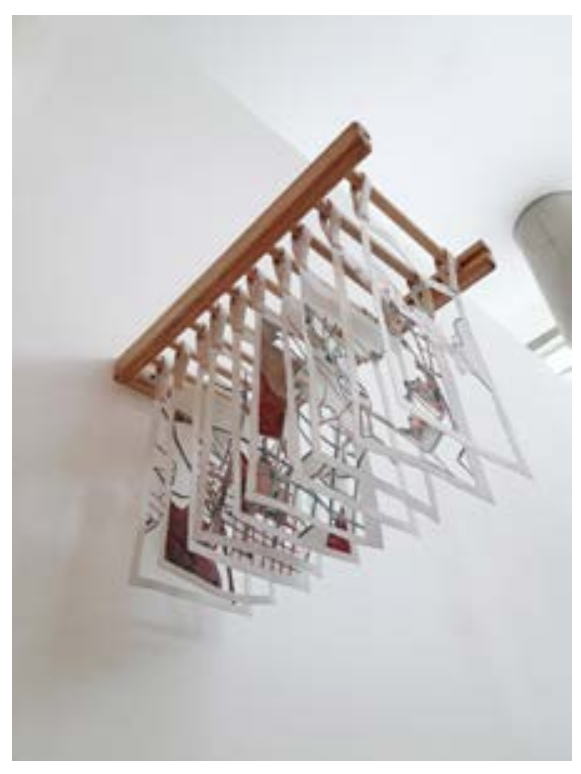

los académicos venezolanos desde el año 2000 hasta el año 2018 a partir de tres subperiodos políticos a saber: 1) la revolución bolivariana de Hugo Chávez (periodo 1999-2006); 2) la construcción del socialismo del siglo XXI de Hugo Chávez (periodo 2007-2012); y 3) la construcción del Estado comunal de Nicolás Maduro (periodo 2013-2018).

El estudio de los salarios de los profesores universitarios requiere de la atención de algunas precisiones: 1) La estructura salarial se organiza, en primer lugar, por los diferentes escalafones de la carrera académica; en segundo lugar, se discrimina en atención a la carga de trabajo que asume el profesor dentro de la gestión académica y universitaria. 2) La escala salarial, es un elemento que vincula la estructura de todos los salarios de los trabajadores y profesores universitarios. En el caso de la estructura de los salarios para los profesores universitarios, se inicia en el escalafón de Instructor a Tiempo Completo (TC). 3) La interescala salarial establece las diferencias monetarias de la escala salarial. Una vez fijado el valor monetario para el salario del Instructor (TC) se procede a aplicar una proporción del aumento del salario a las diversas categorías resultantes de la relación entre el escalafón de la carrera académica y la clasificación de la dedicación del tiempo de trabajo contratado. 4) Los mecanismos para el ajuste salarial estarán determinados por lo establecido en el artículo 13 de las Normas de Homologación. Para ello se procede a tomar el salario base (salario para el profesor DE) y se le aplica el factor de ajuste, tomando en consideración las tasas de inflación de los últimos dos años.

Las anteriores precisiones se complejizan en un contexto económico caracterizado por el alto nivel de la inflación (principal elemento de ajuste, de acuerdo con el artículo 13 de las Normas de Homologación) que ha alcanzado una tasa acumulada de $1940.80 \%$ del IPC entre el 2000 y el 2018 . La reconfiguración de los valores monetarios de los salarios producto de la 
implementación de dos reconversiones monetarias y la presencia de múltiples tasas de cambio (las tasas oficiales y las del mercado paralelo) no permiten presentar los salarios en moneda internacional (dólares americanos) para facilitar una mayor comprensión y análisis del tema.

El ya mencionado contexto configura un escenario de caos financiero que dificulta precisar los efectos económicos de las diferentes actuaciones del gobierno que conducen a la pérdida salarial de los académicos. Nos referimos a las modificaciones que los gobiernos realizaron unilateralmente a la escala salarial, la interescala salarial y el incumplimiento de los mecanismos para el ajuste salarial. Ello obliga a presentar una estimación con cálculos modestos de lo que pudiera ser una aproximación de la pérdida salarial de la cual han sido objeto los profesores universitarios para cada subperiodo. En tal sentido, para la estructura salarial se emplea solo el grupo de profesores clasificados a dedicación exclusiva en todos sus escalafones de la carrera académica; para la escala salarial se toman en consideración los salarios iniciales y finales realmente aprobados por los gobiernos en los diferentes subperiodos de estudio; se calcula la interescala para poder observar la disminución de este valor salarial en cada subperiodo y como mecanismo del ajuste del salario, solo se emplea el índice de inflación acumulado para cada subperiodo. A continuación podremos observar la pérdida salarial monetaria y proporcional para cada subperiodo.

\section{La revolución bolivariana de Hugo Chávez}

Este primer periodo de Hugo Chávez está situado entre 1999 y el 2007, se inicia con su elección como presidente de Venezuela el 6 de diciembre de 1998, en medio de una profunda crisis social, económica y política. El discurso político empleado por Chávez en este periodo fue la refundación de la patria y la instauración del gobierno bolivariano. Entre las acciones políticas de mayor contundencia en esta fase se observa, en primer lugar, la implementación de una Asamblea Constituyente que redactó una nueva norma constitucional, la cual fue aprobada el 15 de diciembre de $1999 .^{2}$ En segundo lugar, en el 2001 Chávez emprende la concentración y centralización de atribuciones no propias del poder ejecutivo mediante la aprobación de la primera Ley Habilitante que dio inicio a confrontaciones que desencadenaron en revueltas sociales y un vacío de poder que tuvo como consecuencia el golpe militar del 2002. En tercer lugar, la denegación del referéndum revocatorio para el cargo de la presidencia de la república en el año 2004. En cuarto lugar, la reelección de Hugo Chávez a su segundo periodo constitucional en diciembre del 2006.

La relación del gobierno bolivariano y la universidad venezolana ha sido tensa y se puede explicar en atención a las políticas públicas aplicadas al sector. Las principales políticas públicas de la educación superior en este primer periodo se suscriben en la implementación de la organización del sistema educativo. En las políticas públicas significativas en materia de organización del sistema de educación superior se encuentran el establecimiento del Viceministerio de Educación Superior, Cultura y Deportes y, posterior a este, el Ministerio de Educación Superior; la implementación del proyecto "Alma Mater"; la creación de las Aldeas Bolivarianas y la mo- 
dificación del mecanismo de acceso a las universidades públicas como antecedentes de lo que a posteriori fue la Misión Sucre y la creación de la Universidad Bolivariana, toda vez que se procuraba posicionar al Estado como órgano rector del nivel educativo (Parra Sandoval, 2015). Finalmente, se agrega la implementación de una política de asfixia presupuestaria contra las universidades que cobra fuerza a partir de 2005, año en el que el Estado venezolano procedió al "rompimiento del mecanismo mixto de financiamiento al sector universitario que se había aplicado desde la década anterior (1995-2005)" (Torres-Núñez, 2016) para establecer un nuevo mecanismo de centralización y diferimiento de los fondos públicos asignados a las instituciones de educación superior (IES).

En este contexto, la política de recuperación salarial -Normas de Homologación- funcionó hasta el bienio 2002-2003, no exenta de luchas y conflictos por parte de los profesores universitarios para hacer valer el contenido de la norma. En efecto, para el bienio 2004-2005, el gobierno impuso un ajuste salarial por la vía del decreto sin discutirlo en el Consejo Nacional de Universidades (CNU) ni con FAPUV, ajuste salarial muy por debajo de la inflación y violentando la escala salarial que regía para la separación de los diferentes escalafones de la carrera académica.

Los datos de la Tabla 1 indican que al finalizar este periodo (bienio 2004-2005) los profesores alcanzaron una pérdida real de su salario que se ubicó entre la proporción del $-20.02 \%$ al $-26.25 \%$, con respecto a los salarios devengados en el bienio 2000-2001. La tasa de inflación acumulada de este subperiodo se ubicó en 107.40\% según los datos publicados por el Banco Central de Venezuela (BCV).

Tabla 1: Estructura salario de los académicos venezolanos 2000-2005

\begin{tabular}{|c|c|c|c|c|c|c|c|c|}
\hline \multirow{3}{*}{$\begin{array}{c}\text { Estructura } \\
\text { salarial }\end{array}$} & \multicolumn{6}{|c|}{ Ajuste salarial } & \multirow{2}{*}{\multicolumn{2}{|c|}{$\begin{array}{c}\text { Pérdida salarial } \\
\text { acumulada } \\
\text { 2000-2005 }\end{array}$}} \\
\hline & \multicolumn{2}{|c|}{ Bienio 2000-2001 } & \multicolumn{2}{|c|}{ Bienio 2004-2005 } & \multirow[b]{2}{*}{ Salario* } & \multirow[b]{2}{*}{ Interescala } & & \\
\hline & Salario base & Interescala & Salario & Interescala & & & Salario & Porcentaje \\
\hline $\begin{array}{c}\text { Instructor } \\
\text { DE }\end{array}$ & $787,570.00$ & & $1,360,950.00$ & & $1,633,420.18$ & & $-272,470.18$ & $-20.02 \%$ \\
\hline $\begin{array}{c}\text { Asistente } \\
\text { DE }\end{array}$ & $968,711.00$ & $22 \%$ & $1,653,109.00$ & $21.50 \%$ & $2,009,106.61$ & $-0.50 \%$ & $-355,997.61$ & $-21.54 \%$ \\
\hline $\begin{array}{c}\text { Agregado } \\
\text { DE }\end{array}$ & $1,191,514.00$ & $22 \%$ & $2,007,828.00$ & $21.50 \%$ & 2,471,200.04 & $-0.50 \%$ & $-463,372.04$ & $-23.08 \%$ \\
\hline $\begin{array}{c}\text { Asociado } \\
\text { DE }\end{array}$ & $1,489,393.00$ & $25 \%$ & $2,478,116.00$ & $23.50 \%$ & $3,089,001.08$ & $-1.50 \%$ & $-610,885.08$ & $-24.65 \%$ \\
\hline $\begin{array}{c}\text { Titular } \\
\text { DE }\end{array}$ & $1,861,741.00$ & $25 \%$ & $3,058,311.00$ & $23.50 \%$ & $3,861,250.83$ & $-1.50 \%$ & $-802,939.83$ & $-26.25 \%$ \\
\hline
\end{tabular}

*Los salarios del Bienio 2004-2005 se ajustaron en 107.40\% de la tasa de IPC acumulada, según los datos publicados por el BCV.

Fuente: Elaboración propia 
Este periodo marca el inicio de lo que en lo sucesivo será la mayor confrontación del gobierno con los académicos venezolanos. La Comisión OPSU-FAPUV no volvió a ser constituida, el instrumento de las Normas de Homologación fue anticonstitucionalmente desconocido por el gobierno y todas las distintas formas de lucha, incluidos paros escalonados e indefinidos y grandes movilizaciones de los universitarios, no lograron preservar la institucionalidad y los necesarios espacios democráticos para la negociación colectiva de sus condiciones de trabajo y remuneración, en un contexto político cada vez más autoritario y un ejercicio gubernamental arbitrario. El ejecutivo nacional con el control de todos los poderes del Estado impuso una política salarial regresiva en franca violación de todas las conquistas laborales del siglo XX venezolano, no solo en las universidades, sino en los distintos sectores laborales del país.

\section{El socialismo del siglo XXI de Hugo Chávez}

Este periodo inicia con la segunda reelección de Hugo Chávez al cargo de la presidencia de la república en enero de 2007 y culmina con la partida oficial de Hugo Chávez a Cuba en diciembre del año 2012. Entre las acciones políticas más relevantes de este periodo podemos señalar, en primer lugar, la profundización de las acciones de nacionalización de las empresas estratégicas, la expropiación de empresas petroleras, la instauración de mayor control de los medios de comunicación y la derrota de la propuesta de modificación de 69 artículos de la constitución que perseguían la implantación del socialismo del siglo XXI en el año 2007. En segundo lugar, la aprobación a través de un referéndum de la enmienda a la constitución para abolir los límites de los periodos para optar a los cargos de elección popular a presidente, gobernadores estatales, alcaldes y diputados a la Asamblea Nacional en el año 2009. En tercer lugar, la aprobación de la Ley Habilitante en el 2010. En cuarto lugar, el nuevo triunfo de Hugo Chávez en las elecciones presidenciales del 2012. Finalmente, Hugo Chávez solo gobierna hasta los primeros días de diciembre del año 2012, cuando parte a Cuba a continuar con su tratamiento médico.

En este periodo la relación entre el gobierno y las universidades fue mucho más tensa, principalmente porque a finales del periodo anterior fueron sustituidos los equipos técnicos del Ministerio de Educación Superior con la firme intención de profundizar el control único del Estado como ente rector del nivel educativo en atención de la refundación de la patria socialista. En este punto resalta la intención del gobierno en modificar el contenido de la autonomía universitaria establecido en la constitución de 1999. Objetivo para el cual el gobierno no solo no tuvo con el apoyo de las universidades públicas tradicionales con largos años de fundación sino que, además, encontró una 
fuerte resistencia de los distintos actores universitarios que hicieron frente a su intención de refundar la autonomía universitaria y facilitar la implementación de la llamada patria socialista dentro de las universidades venezolanas.

A pesar del rechazo popular a la orientación socialista del Estado venezolano, el gobierno de Chávez no dudó en imponer algunos mecanismos que emprendían la implementación antidemocrática de las reformas no aprobadas en el referéndum del año 2007. Nos referimos a las disposiciones contenidas en el Plan Sectorial de Educación Superior 2008-2013, el cual se derivó del Primer Plan Socialista de Desarrollo Económico y Social de la Nación (2007-2013).

Entre las actuaciones de mayor significado en el sector de la educación superior tenemos, en primer lugar, la transformación del antiguo órgano del Ministerio de Educación Superior en Ministerio del Poder Popular para la Educación Superior. Modificaciones que fueron sustantivas en términos de la orientación de su gestión pública -misión y visión- para facilitar la implementación del modelo del socialismo del siglo XXI y la construcción de una sociedad socialista. En segundo lugar, está la precisión del contenido de los objetivos del Plan Sectorial de Educación Superior $2008-2013^{3}$ y el fundamento de un nuevo modelo educativo ${ }^{4}$ como elementos esenciales para el diseño e implementación de las políticas públicas aplicadas en el sector en este periodo. En tercer lugar, se dinamizó la actuación de la Misión Sucre y se implementó una segunda versión del proyecto Alma Mater conceptual y estratégicamente diferente a la primera versión-. En cuarto lugar, se impulsó una nueva institucionalidad ${ }^{5}$ a partir de la transformación de instituciones de educación superior ya existentes y la creación de otras. Finalmente, con esta nueva reconfiguración institucional se impulsó una nueva clase de interlocutores partidistas como mecanismo de neutralización de los legítimos representantes de los actores universitarios, propiciando la presencia de 32 rectores designados en la Asociación de Rectores Bolivarianos (ARBOL), en el Consejo Nacional de Universidades en detrimento político de los 10 rectores agrupados en la Asociación Venezolana de Rectores Universitarios (AVERU) ${ }^{6}$ y la creación de una nueva Federación de Trabajadores Universitarios de Venezuela (FETRAUVE) como interlocutor ilegítimo entre los trabajadores y el gobierno, en perjuicio de la representación legítima de la FAPUV y demás organizaciones gremiales con varias décadas de actuación.

La política salarial de estos bienios estuvo caracterizada por el establecimiento de los decretos unilaterales de ajuste salarial y por el rompimiento de las escalas salariales, las interescalas entre escalafones de la carrera académica y el irrespeto a los lapsos temporales de los bienios, con el propósito de no ajustar los salarios en periodos de al menos tres años. Para el bienio 2006-2007 los ajustes salariales se hicieron al finalizar dicho periodo con 
carácter retroactivo y evidente pérdida del valor real de los mismos por causa de la inflación. Para 2008-2009 se aprobó un ajuste solo para el año 2008 en forma unilateral y contraviniendo el artículo 13 de las Normas de Homologación. No hubo ajuste en 2009. Para el bienio 2010-2011 se aprobó un ajuste unilateral que nuevamente rompió la temporalidad del bienio y no contempló pago para el año 2010 y los meses de enero a abril de 2011, con lo cual el gobierno solo ajustó 8 meses de los 24 que correspondían al periodo del bienio. En año 2012 tampoco hubo ajuste salarial; el salario de los profesores instructores (primer escalafón de la carrera académica) descendió a nivel del salario mínimo -en peores condiciones quedaron los auxiliares docentes, para los cuales el salario quedó por debajo del salario mínimo nacional por varios meses-.

Tabla 2: Estructura salario de los académicos venezolanos 2006-2012

\begin{tabular}{|c|c|c|c|c|c|c|c|c|c|c|}
\hline \multirow{4}{*}{$\begin{array}{c}\text { Estructura } \\
\text { salarial }\end{array}$} & \multicolumn{10}{|c|}{ Pérdida salarial } \\
\hline & \multicolumn{6}{|c|}{ Ajuste salarial } & \multicolumn{2}{|c|}{ Subperiodo } & \multicolumn{2}{|c|}{$\begin{array}{l}\text { Déficit salarial } \\
\text { acumulado }\end{array}$} \\
\hline & \multicolumn{2}{|c|}{ Bienios 2006-2007 } & \multicolumn{4}{|c|}{ Bienio 2010-2011 } & \multirow[b]{2}{*}{ Salario } & \multirow[b]{2}{*}{ Porcentaje } & \multirow[b]{2}{*}{ Salario } & \multirow[b]{2}{*}{ Porcentaje } \\
\hline & Salario & $\begin{array}{l}\text { Inter- } \\
\text { escala }\end{array}$ & Salario & $\begin{array}{l}\text { Inter- } \\
\text { escala }\end{array}$ & Salario* & $\begin{array}{l}\text { Inter- } \\
\text { escala }\end{array}$ & & & & \\
\hline $\begin{array}{c}\text { Instructor } \\
\text { DE }\end{array}$ & 1,831.29 & & 3,335 & & $4,697.26$ & & $-1,362.26$ & $-40.85 \%$ & $-1,634.71$ & $-60.87 \%$ \\
\hline $\begin{array}{c}\text { Asistente } \\
\text { DE }\end{array}$ & $2,205.25$ & $20.50 \%$ & 4,015 & $20.40 \%$ & $5,656.47$ & $-0.10 \%$ & $-1,641.47$ & $-40.88 \%$ & $-1,997.47$ & $-62.42 \%$ \\
\hline $\begin{array}{c}\text { Agregado } \\
\text { DE }\end{array}$ & $2,655.15$ & $20.50 \%$ & 4,834 & $20.40 \%$ & $6,810.46$ & $-0.10 \%$ & $-1,976.46$ & $-40.89 \%$ & $-2,439.84$ & $-63.96 \%$ \\
\hline $\begin{array}{c}\text { Asociado } \\
\text { DE }\end{array}$ & $3,248.32$ & $22.50 \%$ & 5,914 & $22.34 \%$ & $8,331.94$ & $-0.16 \%$ & $-2,417.94$ & $-40.89 \%$ & $-3,028.83$ & $-65.54 \%$ \\
\hline Titular DE & $3,973.36$ & $22.50 \%$ & 7,232 & $22.28 \%$ & $10,191.67$ & $-0.22 \%$ & $-2,959.67$ & $-40.92 \%$ & $-3,762.61$ & $-67.18 \%$ \\
\hline
\end{tabular}

*Los salarios del Bienio 2010-2011 se ajustaron en 156,50\% de la tasa de IPC acumulada, según los datos publicados por el BCV.

Fuente: Elaboración propia

La pérdida salarial de este subperiodo se estimó entre $40.85 \%$ y el $40.92 \%$, y el déficit salarial acumulado para los profesores universitarios venezolanos en este periodo se situó entre $-60.87 \%$ al $-67.18 \%$, lo cual se traduce en menor valor monetario del salario para hacerle frente a la creciente crisis económica expresada, entre otras cosas, en una inflación acumulada del $156.50 \%$ entre el 2006 y el 2012; además, tendríamos que añadir que, pese a la pérdida salarial y los más de tres años sin aplicar ajuste salarial, también se aprecia ya en este periodo las modificaciones hechas a la interescala salarial. Toda esta actuación de atropello contra los profesores universitarios llevó a la radicalización del conflicto entre el gobierno y los académicos que realizaron de una huelga de hambre en el 2011, protagonizada por universitarios, razón por la cual FAPUV interpuso una queja ante la OIT. 


\section{La construcción del Estado Comunal de Nicolás Maduro}

Este periodo inicia en el año 2013 con la elección de Nicolás Maduro a la presidencia de la república, posterior al deceso de Hugo Chávez. La principal oferta electoral durante este periodo ha sido la construcción del Estado Comunal; para ello se ha implementado el Plan de la Patria, ideado por Hugo Chávez. Este periodo está marcado por las crecientes violaciones del orden democrático y constitucional, la profundización de la crisis económica y una creciente conflictividad social; coincidiendo en alguna forma con "la construcción del Estado autoritario" señalado por Parra-Sandoval (2015). Las principales características de este Estado autoritario se sintetizan a continuación.

En primer lugar, las crecientes violaciones al orden democrático y constitucional. La elección de Nicolás Maduro en abril de 2013 tuvo un reducido margen de diferencia electoral (1.49\%) y el cuestionamiento de los resultados electorales por parte de la oposición venezolana, la cual desconoció los resultados ofrecidos por el Consejo Nacional Electoral y procedió a la impugnaron los resultados en el Tribunal Supremo de Justicia. ${ }^{7}$ En el 2015, el partido de gobierno pierde el control de la Asamblea Nacional mientras la oposición conquista 112 curules de 167. Este triunfo le otorgaba a la oposición una amplia fortaleza en el control de las actuaciones del Estado por la posibilidad de administrar las facultades constitucionales que le confiere la ley mediante el control de las dos terceras partes de la Asamblea, situación que encendió una alarma en el gobierno de Nicolás Maduro, que usó extemporáneamente (año legislativo ya culminado) la antigua Asamblea Nacional para proceder la noche del 23 de diciembre a nombrar inconstitucionalmente $^{8}$ a los 34 nuevos integrantes que hoy dirigen el Tribunal Supremo de Justicia. Las actuaciones del nuevo TS han estado fuera del orden constitucional $^{9}$ como la decisión, por parte del CNE, de no celebración del proceso ante la solicitud del referéndum revocatorio al cargo de la presidencia de la república en octubre de 2016; la elección de la Asamblea Nacional Constituyente (ANC) el día 30 de julio de 2017, situación que profundizó la crisis política e institucional y; finalmente, por la vía del decreto emitido por la ANC el día 28 de abril de 2018, se autoriza el adelanto de elecciones para el cargo de la presidencia de la república, en cuyo proceso la oposición venezolana decidió no presentarse.

En segundo lugar, la profundización de la crisis económica. La recesión económica durante el gobierno de Maduro ha estado caracterizada por la súbita caída del precio del petróleo (de $\$ 110$ a menos de $\$ 30$ ) y una inflación bianual del 94\% para los años 2013 y 2014; las diferentes modificaciones del control y administración de las divisas en el Centro Nacional de Comercio Exterior (CENCOEX) el año 2014 y el Sistema de Divisas de Tipo de Cambio Complementario Flotante de Mercado (DICOM) el año 2016; la implementación de la reconversión monetaria en el mes de agosto del año 2018 con el propósito de ocultar la monstruosa devaluación de la moneda nacional, así como la presencia de la mayor tasa de inflación que el país haya conocido, la cual se ubica en $1676.90 \%{ }^{10}$ entre los años 2013 y 2018, según las cifras oficiales publicadas por el BCV. Todo esto explica la destrucción del tejido productivo del país y, con ello, la escasez de alimen- 
tos y medicamentos para la población, elementos fundamentales de lo que ha sido declarada la crisis humanitaria que vive Venezuela.

En tercer lugar, la conflictividad social. Se desconoce el número exacto de las protestas acaecidas durante el periodo de Nicolás Maduro. Han sido muchos años de conflictividad que dejan al menos las siguientes cifras. Según el Foro Penal (2019) la represión social venezolana registrada entre el 01 de enero de 2014 y el 30 de noviembre del año 2018 deja como saldo lo siguiente: 12,949 ciudadanos detenidos arbitrariamente, 7,512 ciudadanos investigados penalmente, la mayoría de ellos con medidas cautelares sustitutivas de libertad (presentación periódica, arresto domiciliario, prohibición para hacer declaraciones a los medios públicos, entre otras medidas coercitivas) y; 812 ciudadanos procesados arbitrariamente por tribunales militares. ${ }^{11} \mathrm{El}$ gobierno de Nicolás Maduro enfrenta graves denuncias de crímenes de lesa humanidad según el informe de la OEA (2018). La grave crisis y el descontento social a inicios del 2014 fueron respondidos por el gobierno con la represión del aparato militar en las manifestaciones populares, las cuales culminaron con al menos 43 muertos y la cárcel del principal dirigente político de oposición para el momento, Leopoldo López; posteriormente, entre el 6 de abril y el 14 de agosto de 2017 se registraron 105 asesinatos en las diferentes protestas sociales; finalmente, quien fuera Fiscal General de la República, la ciudadana Luisa Ortega, denunció ante la Corte Penal Internacional en noviembre del 2017 la cifra de 8,292 ejecuciones extrajudiciales ${ }^{12}$ para el mes de junio de 2017.

En materia de la educación universitaria, el gobierno de Nicolás Maduro consolida el control y centralización de los recursos económicos destinados a las universidades, además de darle continuidad a las políticas de universalidad y modificar discrecionalmente el mecanismo de acceso a las universidades públicas con el discurso de eliminar todos los elementos que generan exclusión económica, cultural y social (Parra-Sandoval, 2015).

La política salarial de estos bienios estuvo marcada por la discusión y aprobación de las tres Convenciones Colectivas del Sector Universitario (ICCU) de los años 2013, 2015 y 2017. En el 2013 se discutió y se aprobó la Convención Colectiva sin la 
participación del gremio de profesores universitarios, al cual se le impuso nuevamente un ajuste salarial que redujo significativamente el valor de las remuneraciones en el sector, por la vía de la inflación. Los derechos del personal jubilado, pensionado y sobreviviente fueron gravemente lesionados en esa primera Convención al introducirse el concepto de pensión indivisible para rebajar las pensiones en comparación con el salario del personal en servicio activo.

Para darle apariencia de negociación a esta política, el gobierno nacional llevó a cabo las negociaciones con FETRAUVE, la organización sindical que ya había sido creada, conformada por el partido de gobierno, con la anuencia del Ministerio del Trabajo. Esta organización no logra agrupar ni siquiera a un $10 \%$ de los universitarios, incluidos empleados y obreros, pero fue la que reconoció el gobierno en abierta violación a la libertad sindical. Estas violaciones recurrentes a derechos adquiridos, a la libertad sindical y al derecho constitucional a convenir las condiciones de remuneración y trabajo de los profesores universitarios provocaron el conflicto y fueron la causa del paro nacional de 2013, que se resolvió con un acuerdo suscrito entre FAPUV, la Asociación Venezolana de Rectores Universitarios (AVERU) y el Ministerio, en el que se reconoce a la FAPUV como legítima representante de los profesores universitarios; se ratifica la progresividad e intangibilidad de los derechos laborales de los profesores universitarios y se reconoce la vigencia de los acuerdos federativos (Normas de Homologación) y Actas Convenio Institucionales suscritas entre las universidades y sus asociaciones de profesores.

En este escenario de centralización y diferimiento de los fondos públicos asignados a las IES por el Estado cada vez más autoritario se profundiza, como hemos visto, la destrucción del salario de los académicos y a partir de 2013, la sustitución unilateral e ilegal de las Normas de Homologación por la implementación de tres contrataciones colectivas (años 2013-20152017). Estas contrataciones dejaron de lado el concepto de indexación salarial y terminaron de destruir las remuneraciones y beneficios en el sector.

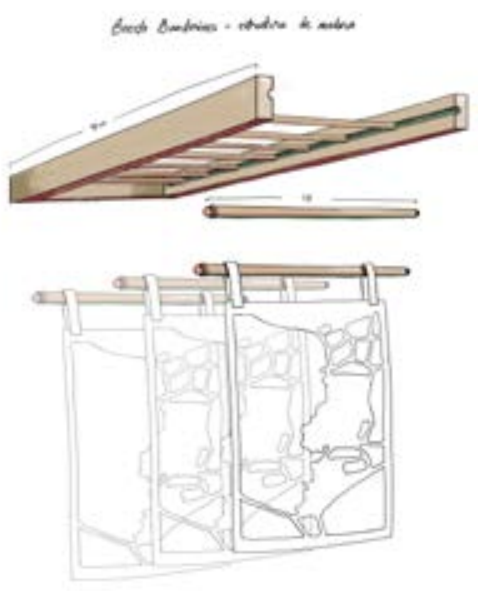

Universidades $\mid$ núm. 83, enero-marzo 2020 | UDUAL | DOI:https://doi.org/10.36888/udual.universidades.2020.83.79 
Tabla 3: Estructura salario de los académicos venezolanos 2013-2018

\begin{tabular}{|c|c|c|c|c|c|c|c|c|c|c|}
\hline & \multicolumn{4}{|c|}{ Estructura salarial } & \multicolumn{2}{|c|}{ Ajuste salarial } & \multicolumn{2}{|c|}{$\begin{array}{l}\text { Pérdida salarial } \\
\text { subperiodo }\end{array}$} & \multicolumn{2}{|c|}{$\begin{array}{l}\text { Déficit salarial } \\
\text { acumulado }\end{array}$} \\
\hline & \multicolumn{2}{|c|}{ Bienios 2013-2018 } & \multicolumn{2}{|c|}{ Bienio 2010-2011 } & & & & & & \\
\hline & Salario* & $\begin{array}{l}\text { Inter- } \\
\text { escala }\end{array}$ & Salario** & $\begin{array}{l}\text { Inter- } \\
\text { escala }\end{array}$ & Salario & $\begin{array}{l}\text { Inter- } \\
\text { escala }\end{array}$ & Salario*** & Porcentaje & Salario & Porcentaje \\
\hline $\begin{array}{c}\text { Instructor } \\
\text { DE }\end{array}$ & 6,283 & & $6,381.8$ & & $111,642.63$ & & $-105,260.83$ & $-1,649.39 \%$ & $-106,895.54$ & $-1,710.26 \%$ \\
\hline Asistente DE & 7,345 & $16.90 \%$ & $6,381.8$ & $0 \%$ & $130,513.31$ & $-16.90 \%$ & $-124,131.51$ & $-1,945.09 \%$ & $-126,128.97$ & $-2,007.50 \%$ \\
\hline $\begin{array}{c}\text { Agregado } \\
\text { DE }\end{array}$ & 8,491 & $15.60 \%$ & $7,019.98$ & $10 \%$ & $150,876.58$ & $-5.60 \%$ & $-143,856.60$ & $-2,049.25 \%$ & $-146,296.44$ & $-2,113.21 \%$ \\
\hline Asociado DE & 10,178 & $19.90 \%$ & $7,658.16$ & $9.09 \%$ & $180,852.88$ & $-10.81 \%$ & $-173,194.72$ & $-2,261.57 \%$ & $-176,223.55$ & $-2,327.11 \%$ \\
\hline Titular DE & 12,238 & $20.23 \%$ & $7,658.16$ & $0 \%$ & $217,457.02$ & $-20.23 \%$ & $-209,798.86$ & $-2,739.55 \%$ & $-213,561.47$ & $-2,806.73 \%$ \\
\hline
\end{tabular}

*La expresión monetaria es en bolívares fuertes, los cuales representan 1000 bolívares antes de la reconversión monetaria 2008.

**La expresión monetaria es en bolívares soberanos, representan 100, 000 bolívares fuertes antes de las tres reconversiones monetarias.

***Se aplicó el 1676.90\% de ajuste a los salarios como tasa de IPC acumulada, según los datos publicados por el Banco Central de Venezuela.

Fuente: Elaboración propia

Como resultado del gobierno de Nicolás Maduro y la aplicación de las contrataciones colectivas se estima una pérdida salarial de este subperiodo entre $-1,649.39 \%$ y $-2,739.55 \%$ del salario de los profesores universitarios, con respecto al ajuste que correspondía si se aplicaran los mecanismos establecidos en las Normas de Homologación, tomando como principal referencia la tasa de inflación que alcanzó a ser 1,676.90\% en este subperiodo. Asimismo, se observa la destrucción de la interescala salarial e incluso en este periodo se modificó la estructura salarial de los obreros y los empleados, situación que también significó menores recursos para la estimación del salario base de los profesores universitarios, ${ }^{13}$ el cual sumó un déficit de pérdida salarial que osciló entre una proporción del -1,710.26\% y $-2,806.73 \%$ con respecto a los salarios devengados en el 2001.

\section{Precisión de la crisis humanitaria de los profesores universitarios}

Los salarios de los académicos venezolanos son complejos de estudiar, cada subperiodo en estudio tuvo sus razones políticas, económicas y sociales particulares que impactaron en la calidad de vida de los profesores universitarios. Entre los elementos de mayor relevancia que impiden precisar los cálculos de la pérdida de capacidad salarial se encuentran los altos índices de inflación, los efectos monetarios de las dos reconversiones monetarias y la imposibilidad de expresar los salarios en dólares. Sin embargo, el Sistema de Divisas de Tipo de Cambio Complementario Flotante de Mercado (DICOM) que entró en vigor en enero de 2018 es el mecanismo de divisas fluctuante que ha permitido la flexibilización en la liberación del precio del dólar. 
En tal sentido, presentamos una breve comparación de los salarios del año 2001 y finales del 2018 expresados en dólares con el propósito de brindar más información que legitime la que ha sido la política de destrucción de la profesión académica mediante el desgaste salarial.

Tabla 4: Salario de los académicos venezolanos expresados en dólares para el año 2001 y 2018

\begin{tabular}{|c|c|c|c|c|c|c|c|}
\hline & \multicolumn{4}{|c|}{ Estructura salarial } & \multicolumn{2}{|c|}{ Pérdida salarial } & \multirow{3}{*}{ 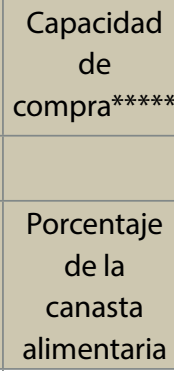 } \\
\hline & \multicolumn{2}{|c|}{ Salario 2001} & \multicolumn{2}{|c|}{ Salario 2018} & & & \\
\hline & Salario* Bs. & Salario* $\$$ & Salario** & $\begin{array}{c}\text { Salario }^{* * *} \\
\$\end{array}$ & $\begin{array}{c}\text { Salario } \\
\mathrm{S}^{* * * *}\end{array}$ & Porcentaje & \\
\hline Instructor DE & $787,570.00$ & 1039.01 & 6275 & 9.83 & $-1.029,18$ & $-99.05 \%$ & $13,95 \%$ \\
\hline Asistente DE & $968,711.00$ & 1277.98 & 6653 & 10.42 & -1267.56 & $-99.18 \%$ & $14,79 \%$ \\
\hline Agregado DE & $1,191,514.00$ & 1571.92 & 7055 & 11.05 & -1560.86 & $-99.30 \%$ & $15,69 \%$ \\
\hline Asociado DE & $1,489,393.00$ & 1964.90 & 7490 & 11.74 & -1953.16 & $-99.40 \%$ & $16,61 \%$ \\
\hline Titular DE & $1,861,741.00$ & 2456.12 & 7928 & 12.42 & -2443.70 & $-99.49 \%$ & $17,63 \%$ \\
\hline
\end{tabular}

*La moneda en vigencia era el bolívar. La reconversión monetaria del año 2008 fijó 1000 bolívares por cada unidad de bolívar fuerte.

**La moneda en vigencia es el bolívar soberano. Luego de tres reconversiones monetarias equivalía a 100.000 bolívares fuertes.

***Se aplicó el valor de la divisa oficial que para diciembre del año 2001 era de 758 bolívares por 1 dólar según el BCV.

****Se aplicó el valor de la divisa oficial, para diciembre del año 2018 era de bolívares soberanos 638.18 por 1 dólar según el BCV.

*****El costo de la Canasta Alimentaria Básica se situó en 44954.13 bolívares soberanos, equivalente a 70.44 dólares.

Fuente: Elaboración propia, en base a la reconstrucción de los datos publicados por Muñoz (2019).

Los datos contenidos en la tabla anterior evidencian la implementación de la política de destrucción y aniquilamiento de la profesión académica en todos los subperiodos de estudio. Es injustificable que el gobierno venezolano hoy esté hurtando de manera despiadada más del $99 \%$ del salario en dólares que les correspondería a los académicos venezolanos, para situarlos en un estado de miseria que les impide la subsistencia a ellos y a su núcleo familiar, toda vez que ni siquiera logran comprar los insumos de la canasta alimentaria, como se evidencia en la Tabla número 4.

Razón suficiente para que la Asamblea Nacional, en septiembre de 2018, haya decidido aprobar un acuerdo para declarar la "Emergencia Humanitaria Compleja de la Educación en Venezuela”. En el mismo documento se ex- 
horta al gobierno a que atienda el mandato de sus responsabilidades constitucionales, sobre todo lo relativo al ámbito educativo, con el propósito de procurar una educación de calidad. Esta declaración generó una reacción de apoyo y adhesión al contenido del acuerdo por parte de varios Consejos Universitarios de las universidades mayormente agrupadas en AVERU y la FAPUV.

Sin lugar a duda, la destrucción del salario y, en consecuencia, de la profesión académica conduce al deterioro de la actividad científica. En el caso de Venezuela, esto ha sido particularmente grave debido al cerco económico impuesto por el gobierno a las universidades, con presupuestos centralizados por el Ministerio, cuyo valor ha disminuido de manera sostenida y en los cuales, el gobierno ha llevado a su mínima expresión la cuota que se destina a la actividad de investigación científica.

Como consecuencia, la producción científica en las universidades venezolanas y las publicaciones de nuestros investigadores en revistas indizadas y arbitradas en el mundo han disminuido. De la misma manera, la producción de revistas científicas venezolanas se ha visto gravemente afectada y en su mayoría han tenido que migrar al formato digital y modificar su periodicidad para poder mantenerse (Hernández, 2016). A esto hay que agregar el éxodo de investigadores con publicaciones y producción científica permanente que esta situación ha generado, lo cual ha conllevado a que en los últimos años alrededor de 500 investigadores al año han emigrado y otra gran proporción se desplaza a otros oficios que le permitan subsistir económicamente (Requena, 2016).

Finalmente, el colapso de la universidad venezolana es el resultado de tres lustros de una desinversión programada por parte del gobierno, de una política salarial y presupuestaria regresiva y continuada que hoy impide que se cumpla para todos el derecho constitucional a la educación universitaria gratuita y de calidad, que ha desmantelado la investigación científica, que somete, atropella, persigue y condena a los académicos que aún resisten con dignidad dentro de las universidades venezolanas, al tiempo que ha desplazado a gran parte de los profesores universitarios a otros sectores de la economía nacional e incluso a otros países, donde buscan desarrollarse -en su actividad académica o no- en condiciones adecuadas y percibir una remuneración acorde con su talento y esfuerzo. 


\section{Notas}

1. Así, en la medida en que las condiciones económicas del país han desmejorado, las Normas de homologación han llegado a ser consideradas por los profesores universitarios como un mecanismo de "recuperación del salario".

2. El contenido político de la nueva constitución fue fundamental para desencadenar los otros tres eventos señalados en este punto. Algunos de los elementos más significativos fue la extensión del período presidencial, la consulta popular para revocatorios de los cargos públicos, la reelección inmediata, la eliminación bicameral del congreso por el un poder legislativo unicameral, además, se restructuraron y cambiaron los nombres de los otros poderes públicos.

3. Los objetivos de mayor relevancia fueron: universalizar la educación superior, fortalecer las capacidades para la generación y socialización de conocimientos, impulsar un nuevo modelo educativo, crear nuevas instituciones de educación superior y transformar las existentes.

4. El nuevo modelo educativo estaría centrado en la formación ética, la transformación social, la comprensión de nuevas realidades, entornos y producción socialista.

5. Básicamente la nueva institucionalidad fue la transformación a universidades experimentales de 29 institutos y colegios universitarios oficiales; creación de 17 universidades territoriales y 10 especializadas y 2 institutos especializados; la creación de la universidad de los trabajadores y otra de los pueblos del sur; y la creación de los Complejos Universitarios Socialistas Alma Mater (CUSAM).

6. Asociación que agrupa a los Rectores de las cinco universidades autónomas y otras cinco con autonomía limitada.

7. La Mesa de la Unidad Democrática solicitó una auditoria del proceso al Consejo Nacional Electoral, este órgano declaró que, por ser automatizado el proceso, la auditoría propuesta por la oposición no se podía realizar en los términos señalados y procedió a fijar el mecanismo para la revisión. La oposición venezolana impugnó las elecciones presidenciales, las cuales el Tribunal Supremo de Justicia, el día 07 de agosto del mismo año, dictaminó inadmisibles.

8. Se violó el Artículo 264 de la Constitución (1999) y el Título V de la Ley Orgánica del Tribunal Supremo de Justicia (LOTSJ), con relación al cumplimiento del lapso requeridos (30 días continuos) para realizar el proceso para la designación de los magistrados, tanto principales como suplentes. No hubo lugar para conformar un legítimo Comité de Postulaciones Judiciales que hiciera una primera preselección de candidatos y luego se remitiera al Ministerio Público, Defensoría del Pueblo y Contraloría General de la República, además el proceso contempla una segunda preselección de los candidatos a los cargos, para luego ser considerada por la Asamblea Nacional en un lapso de tres sesiones plenarias para escoger a los magistrados mediante el voto de las $2 / 3$ partes de los diputados o una cuarta sesión plenaria para la elección por voto de mayoría simple en caso de no lograrse con el voto de las $2 / 3$ partes de la AN. El proceso para la selección de los 13 magistrados principales y los 21 magistrados suplentes se llevó a cabo la noche del 23 de diciembre de 2015, con el atenuante que el año legislativo había concluido el 15 de diciembre de 2015.

9. Entre las actuaciones de mayor significado político están la suspensión de la proclamación de 4 diputados del Estado Amazonas el día 30 de diciembre de 2015, con la firme intención de vulnerar las futuras actuaciones y decisiones constitucionales facultadas a las dos terceras partes de la Asamblea Nacional recién electa. El decreto de suspensión de todos los actos administrativos de la Asamblea Nacional, emitido en enero de 2016; delegación de las facultades de la Asamblea Nacional en el poder ejecutivo mediante decretos, para que ejerza su gestión sin ningún control y la decisión del TSJ de asumir como propios los poderes de la Asamblea nacional 155 y 156, emitidas el 29 de marzo de 2017.

10. El Banco Central de Venezuela publicó las siguientes tasas de los índices de precios al consumidor IPC para el periodo presidencial de Nicolás Maduro. Año 2013 - 43\%; año 2014 - 50\%; año 2015 - 99.6\%; año 2016 - 148.6\%; año 2017 - 271\%; año 2018 - 1063.6\%.

11. Los datos actualizados para el 30 de septiembre de 2019 demuestran el incremento del autoritarismo del gobierno de Nicolás Maduro: 15,170 ciudadanos detenidos por asuntos políticos, 470 presos políticos, 8,097 ciudadanos con medidas cautelares de libertad y 848 procesados en tribunales militares (Foro Penal, 2019).

Universidades $\mid$ núm. 83, enero-marzo 2020 | UDUAL $\mid$ DOI:https://doi.org/10.36888/udual.universidades.2020.83.79 
12. Número total de personas asesinadas por funcionarios policías y militares (OEA, 2018; p. 61).

13. Recordamos que el cálculo base para el salario de los profesores se vinculaba al salario del segundo nivel del profesional.

\section{Referencias}

Centro de Documentación y Análisis para los Trabajadores (2019). Canasta alimentaria. Noviembre 2018. Disponible en: http://cenda.org.ve/noticia.asp?id=181

Foro Penal (2018). Reporte sobre la represión en Venezuela. Noviembre 2018. Caracas. Disponible en: https://foropenal.com/reporte-sobre-la-represion-en-venezuela-noviembre-2018/

Foro Penal (2019). Reporte sobre la represión en Venezuela. Septiembre 2019. Caracas. Disponible en: https://foropenal.com/reporte-sobre-la-represion-en-venezuela-septiembre-2019/

Hernández Fernández, L. (2016). Presupuesto universitario deficitario y la vigencia de la revistas científicas. Revista de Ciencias Sociales (Ve), vol. XXII, núm. 1, enero-marzo, 2016, pp. 7-8 Universidad del Zulia Maracaibo, Venezuela Disponible en: https://www.redalyc.org/ pdf/280/28046365001.pdf

Muñoz, A. (@AlvaroMunoz) “¡ACTUALIZADA! Tablas de sueldos con el ajuste de sueldo impuesto por el "tren patronal”, a tasa del BCV del viernes 09/08/19. @FAPUV @OITAmericas \#UniVE \#Venezuela @ReporteYa \#11/08/19 @UCLAve @mbachelet” agosto 11, 2019, 10:36 p.m., [Tuit]. <https://mobile.twitter.com/alvaromunoz/status/1160741779920101377?s=12> [consulta: 12 agosto 2019]

Organización de Estado Americanos (2018). Informe de la Secretaría General de la Organización de los Estados Americanos y del Panel de Expertos Internacionales Independientes sobre la posible comisión de crímenes de lesa humanidad en Venezuela / Secretaría General de la Organización de los Estados Americanos. Washington DC. Disponible en: http://www.oas. org/documents/spa/press/Informe-Panel-Independiente-Venezuela-ES.pdf

Parra, M. C. (1998). Análisis de algunos indicadores de la educación superior en Venezuela. Cuadernos del CENDES, ISSN 1012-2508, No. 37 (enero-abril), pp. 221-244.

Parra-Sandoval, M. C. (2003). La profesión académica en Venezuela de los «catedráticos» a los profesores universitarios. Cuadernos del CENDES, ISSN 1012-2508, № 53 (mayo-agosto), pp. 93-120.

Parra Sandoval, M. C. (2015). Venezuela: las políticas de educación superior en el proceso revolucionario, Propuesta Educativa, núm. 43, junio, 2015, pp. 88-98. Facultad Latinoamericana de Ciencias Sociales. Buenos Aires, Argentina. Disponible en: http://www.redalyc.org/ articulo.oa? $\mathrm{id}=403041714009$

Requena, J.; Caputo, C. (2016). Pérdida de talento en Venezuela: migración de sus investigadores. Caracas: Interciencia, vol. 41, núm. 7, julio, 2016, pp. 444-453.

Torres-Núñez, Luis E. (2016). Criterios a considerar para la financiación de la educación superior en Venezuela. Revista Internacional de Educação Superior v. 2 n. 3, ISSN: 2446-9424. Campinas SP: Universidade Estadual de Campinas. Disponible en: https://periodicos.sbu.unicamp.br/ ojs/index.php/riesup/article/view/8650561 